\title{
Shrinkage Control of Photoresist for Large-Area Fabrication of Sub-30 nm Periodic Nanocolumns
}

\author{
Hai Le-The,* Erwin Berenschot, Roald M. Tiggelaar, Niels R. Tas, Albert van den Berg, \\ and Jan C. T. Eijkel*'
}

\begin{abstract}
A method to fabricate large-area arrays of nanocolumns without a deep-UV laser source is reported. This method allows high-yield fabrication of $3 \times 3 \mathrm{~cm}^{2}$ arrays of sub- $30 \mathrm{~nm}$ nanocolumns made of bottom antireflection layer coating (BARC) by combining displacement Talbot lithography (DTL) using a monochromatic UV beam ( $365 \mathrm{~nm}$ wavelength) with plasma etching techniques. DTL is used to manufacture an initial pattern of periodic photoresist nanocolumns with a diameter of $\approx 110 \mathrm{~nm} . \mathrm{N}_{2}$ plasma can transfer this pattern at a 1:1 ratio to BARC nanocolumns. It is found that reactive $\mathrm{O}_{2} / \mathrm{N}_{2}$ plasma etching on the other hand can shrink the BARC nanocolumns to sub-30 nm dimensions. The shrink-etching process can be reproducibly controlled by tuning the gas flow ratio and the etching time. It is highly remarkable that the verticality of these BARC nanocolumns remains during $\mathrm{O}_{2} / \mathrm{N}_{2}$ plasma shrink etching. Combining the etching of $\mathrm{O}_{2} / \mathrm{N}_{2}$ plasma with $\mathrm{N}_{2}$ plasma allows to produce BARC nanocolumns over the entire diameter range from 110 to sub-30 $\mathrm{nm}$. The fabrication approach enables largefootprint fabrication of size-tunable periodic nanostructures that have many potential applications in photonics, electronics, biosensors, smart surfaces, catalysis, and biomedical analysis.
\end{abstract}

\section{Introduction}

Nanotechnology in recent decades has provided opportunities to synthesize nanomaterials and to pattern nanostructures. The enormous increase in nanotechnology applications in physics, chemistry, biological/medical applications, and in

H. Le-The, Prof. A. van den Berg, Prof. J. C. T. Eijkel BIOS Lab-on-a-Chip Group

MESA+ Institute for Nanotechnology

MIRA Institute for Biomedical Technology

and Technical Medicine

University of Twente

7522 NB, Enschede, The Netherlands

E-mail: h.lethe@utwente.nl; j.c.t.eijkel@utwente.nl

E. Berenschot, Dr. N. R. Tas

Mesoscale Chemical Systems Group

MESA+ Institute for Nanotechnology

University of Twente

7522 NB, Enschede, The Netherlands

Dr. R. M. Tiggelaar

NanoLab Cleanroom

MESA+ Institute for Nanotechnology

University of Twente

7522 NB, Enschede, The Netherlands

DOI: $10.1002 / \mathrm{admt} .201600238$ materials science leads to the requirement for robust, high-yield fabrication techniques to precisely pattern nanostructures over large areas and at low cost. ${ }^{[1,2]}$ Near-UV-based optical lithography techniques such as projection lithography and proximity lithography allow to pattern structures in the sub-micrometer range but are limited to $\approx 0.35 \mu \mathrm{m}$ feature size. ${ }^{[3]}$ Fabrication using these techniques to achieve such critical dimension requires dedicated systems, which are difficult to operate reproducibly. Several techniques have been successfully utilized to meet the great demand for surface patterning at the nanoscale, including electron beam lithography $(\mathrm{EBL}),{ }^{[4,5]}$ ion beam lithography $(\mathrm{IBL}){ }^{[6]}$ laser interference lithography (LIL), ${ }^{[7-9]}$ nanoimprint lithography, ${ }^{[10,11]}$ and nanosphere lithography. ${ }^{[11-14]}$ EBL and IBL provide the opportunity to produce feature sizes as small as $10 \mathrm{~nm}$ but require conducting substrates and delicate operating systems. Moreover, because of their low throughput they are not suitable for batch production. LIL-with the use of the interference of two coherent laser beams - can produce periodic nanopatterns down to $\approx 20 \mathrm{~nm}$ over large areas. Although featuring high-yield fabrication, this technique requires high system stability in order to achieve reproducible fabrication. Nanoimprint lithography is also suitable for large-footprint nanopatterning at low cost; however, it requires flat substrates and an additional step to remove the residual polymer layer. Nanosphere lithography relies on the self-assembly of colloidal spheres, but this leads to a lack of control and hence is not applicable for large-area fabrication of periodic nanostructures.

Another direct way to decrease the feature size is to decrease the wavelength of the illumination sources. Using a laser source with a shorter exposure wavelength, e.g., 157 or $193 \mathrm{~nm}$, photolithography could possibly produce sub-50 $\mathrm{nm}$ features..$^{[15]}$ Using extreme ultraviolet lithography interferometry with a synchrotron source wavelength of $13 \mathrm{~nm}$, the fabrication of $19 \mathrm{~nm}$ lines and spaces, and $38 \mathrm{~nm}$ period gratings has been demonstrated. ${ }^{[16,17]}$ Although these techniques can provide excellent resolution enhancement in downscaling the feature size, they require high cost and complex operating systems that are not widely available.

Recently, Talbot lithography has been used as an alternative for patterning high-resolution periodic nanofeatures. ${ }^{[18-20]}$ 


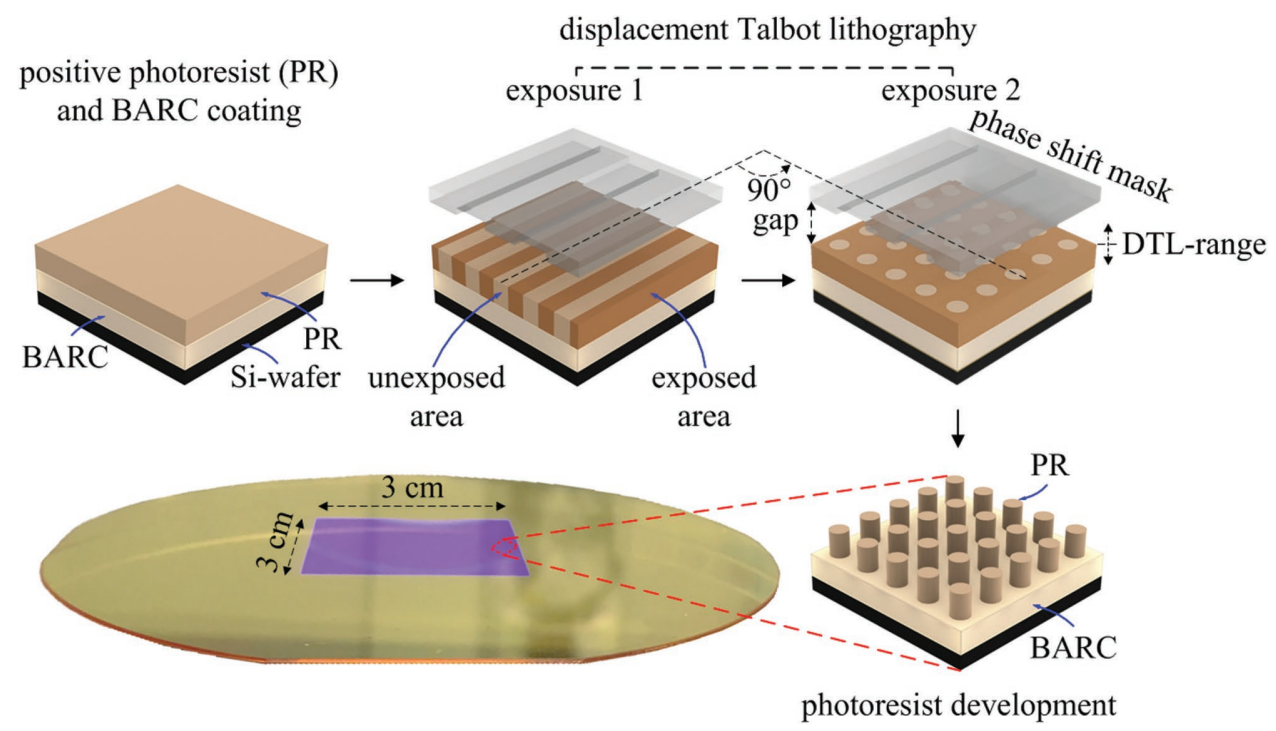

Figure 1. Fabrication process of a $3 \times 3 \mathrm{~cm}^{2}$ array of $P R$ nanocolumns using DTL.

This technique relies on the near-field diffraction effect that was first observed by Talbot. ${ }^{[21]}$ When illuminating a periodic pattern (grating) with monochromatic light, its image is repeated at periodic distances away from the grating plane. This distance, termed the Talbot distance, is proportional to both the square of the grating period and the reciprocal of the wavelength. ${ }^{[22]}$ Although effective, Talbot lithography is limited by the very small Talbot distances of the self-images, which requires extremely flat substrates and precise control of the distance from the mask to the substrate. Significant progress was reported by Solak et al. with the introduction of an improved version of this technique, termed displacement Talbot lithography (DTL). ${ }^{[23]}$ During the illumination of the mask using a monochromatic UV beam (365 nm wavelength), after a period of time the substrate is displaced within a DTL range around a set gap distance from the mask. The substrate is then scanned through the self-images of the predefined periodic structures on the mask at every Talbot distance. Therefore, this effective technique could provide rapid wafer-level patterning of highresolution periodic nanostructures, i.e., regular patterns of lines, holes, or dots in photoresist (PR), without the need for flat substrates nor an absolute/fixed distance between the mask and the substrate. For the high-resolution patterning of photoresist nanostructures using this technique, a bottom antireflection layer coating (BARC) is required. BARC is used to absorb the light passing through the photoresist layer to reduce the interference effect caused by the reflection of light on the Siwafer surface. ${ }^{[24,25]}$ Despite being a powerful nanopatterning technique, UV-based DTL is limited in its application to nanostructures of hundreds of nanometers. Thus, patterning sub$100 \mathrm{~nm}$ periodic nanostructures by applying the DTL technique then still requires a deep-UV laser, i.e., an ArF laser operating at $193 \mathrm{~nm}$.

In this paper, we report an alternative robust method to fabricate sub-100 $\mathrm{nm}$ periodic nanocolumns using the DTL technique but without the need for a deep-UV laser source. Combining DTL using a UV beam source (365 nm wavelength) with plasma etching techniques enables us to produce peri- odic nanocolumns made of BARC at various sub-100 nm diameters from the predefined $110 \mathrm{~nm}$ photoresist nanocolumns patterned by DTL. We have demonstrated the fabrication of large-area arrays of BARC nanocolumns at diameters ranging from 110 to sub-30 nm, by combining various plasma etchings $\left(\mathrm{N}_{2}, \mathrm{O}_{2}\right.$, and $\mathrm{O}_{2} / \mathrm{N}_{2}$ plasmas) and by tuning the etching parameters such as $\mathrm{O}_{2} / \mathrm{N}_{2}$ gas flow ratio and varying the etching time. Using our fabrication approach, well-defined BARC nanocolumns with diameters as small as $28 \mathrm{~nm}$ and high aspect ratios up to 5.3 over large areas of $3 \times 3 \mathrm{~cm}^{2}$ were reproducibly fabricated with high uniformity.

\section{Results and Discussion}

\subsection{Patterning Photoresist Nanocolumn Arrays}

Details of the fabrication process are given in the Experimental Section. A schematic of the fabrication process for PR nanocolumn arrays is shown in Figure 1. Starting with a silicon (Si) wafer, the wafer without its native oxide is coated with a BARC of $186 \pm 2 \mathrm{~nm}$, and then with a $200 \pm 3 \mathrm{~nm}$ positive PR layer. Displacement Talbot lithography ${ }^{[23]}$ is used to pattern the periodic PR nanocolumns by two exposures using a phase shift mask, and between both exposures the wafer is rotated by $90^{\circ}$ with respect to the mask. The phase shift mask consists of a linear grating with a period of $500 \mathrm{~nm}$ over the large area of $3 \times 3 \mathrm{~cm}^{2}$ etched into a fused-silica substrate to provide an $\approx 180^{\circ}$ phase shift between the light transmitted through the lines and the spaces. As a result, a strong Talbot effect can be obtained at an suitable distance from the mask. ${ }^{[23]}$ After developing the exposed $\mathrm{Si}$ wafers, the exposed areas of PR are stripped away, leaving $3 \times 3 \mathrm{~cm}^{2}$ arrays of PR nanocolumns at the center of the Si wafers.

Figure 2a shows high-resolution scanning electron microscope (HR-SEM) images of the fabricated PR nanocolumn array. Well-defined PR nanocolumns were obtained that had vertical sidewalls as shown in the cross-sectional image. The 

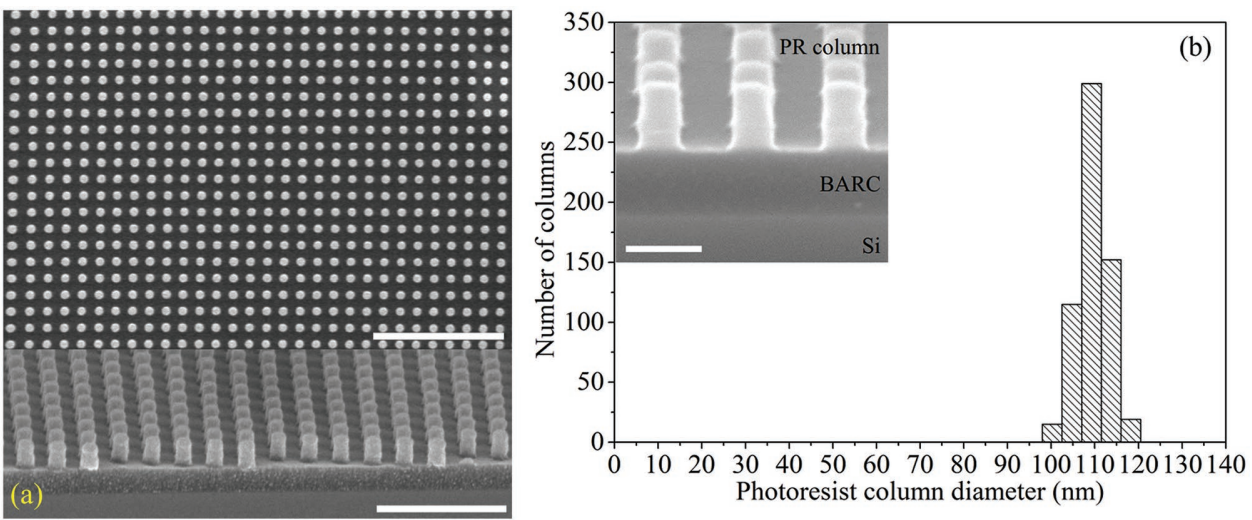

Figure 2. a) Top-view (scale bar: $2 \mu \mathrm{m}$ ) and cross-sectional (scale bar: $1 \mu \mathrm{m}$ ) HR-SEM images of periodic PR nanocolumns patterned by DTL. b) Diameter distribution of the PR nanocolumns with a close-up image inserted (scale bar: $200 \mathrm{~nm}$ ).

top-view HR-SEM image shows a well-controlled pattern of PR nanocolumns (250 $\mathrm{nm}$ pitch) with a high uniformity in terms of diameter distribution (Figure $2 \mathrm{~b}$ ). The diameter of the PR nanocolumns was $\approx 110 \mathrm{~nm}$ with a small variation (1 standard deviation) of $3 \mathrm{~nm}$. Five selected areas over the $3 \times 3 \mathrm{~cm}^{2}$ array were measured and showed well-fabricated and well-controlled PR nanocolumns, though the diameter varies slightly, i.e., $4.7 \mathrm{~nm}$ (see Figures S1 and S2, Supporting Information). This could be due to imperfections in the used mask (i.e., a replica of a mask made by electron beam lithography) which were transferred directly into the PR patterns. Additionally, the loss of focus and drifting of the HR-SEM images ${ }^{[26]}$ during a slow scan speed could also add to some inaccuracy during image processing and analysis by using Image J software. Overall these images indicate a robust fabrication process.

\subsection{Plasma Etching of Bottom Antireflection Layer Coating}

To subsequently transfer the fabricated PR nanocolumn arrays into the BARC layer, plasma etching was chosen as a suitable technique. Plasma etching is considered as an excellent approach for transferring PR structures patterned from the lithography process to BARC layers because of its high etchrate selectivity for BARC over PR. With a minimum resist loss, it thus allows further processing the substrates. ${ }^{[27,28]} \mathrm{A}$ reactive

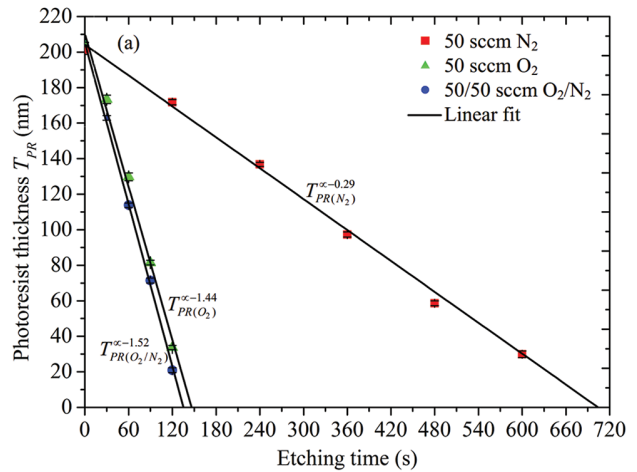

ion etch system (home-built TEtske machine, NanoLab) was used to conduct all our etching processes (see Figure S3, Supporting Information).

We first investigated the etching rate of unpatterned PR and BARC layers in the TEtske machine using various gas plasmas, i.e., $50 \mathrm{sccm} \mathrm{N}_{2}$ plasma, $50 \mathrm{sccm} \mathrm{O}_{2}$ plasma, and 50/50 $\mathrm{sccm}$ $\mathrm{O}_{2} / \mathrm{N}_{2}$ plasma, all at $13 \mathrm{~m}$ Torr and $25 \mathrm{~W}$. The etching was conducted at the wafer level in a clean etching chamber in order to prevent the loading effect and the redeposition of residual materials deposited on the chamber wall from the previous processes (see Figure S4, Supporting Information). Figure 3 shows the measured thicknesses of the PR and BARC layers on the Si wafers versus the etching time. For all gas plasmas, a linear decrease in the thicknesses of the PR and BARC layers was observed with increasing etching time, as evidenced by the almost perfect linear fit curves (see Equations (S1)-(S6), Supporting Information). The etching rates of the PR and BARC found at these etching settings are shown in Table 1.

A considerable etch-rate selectivity of BARC over PR (1.34) was achieved in $50 \mathrm{sccm} \mathrm{N}_{2}$ plasma. This significant difference in etching rates was also reported by Gupta et al, ${ }^{[27]}$ who attributed it to the difference in molecular structure between BARC (AZ BARLi) and PR (i-line photoresist). ${ }^{[27]}$ The i-line photoresist is a novolac (phenol-formaldehyde)-based resist containing aromatic rings, methyl and hydroxyl groups, whereas the molecular structure of the BARC contains oxygen atoms

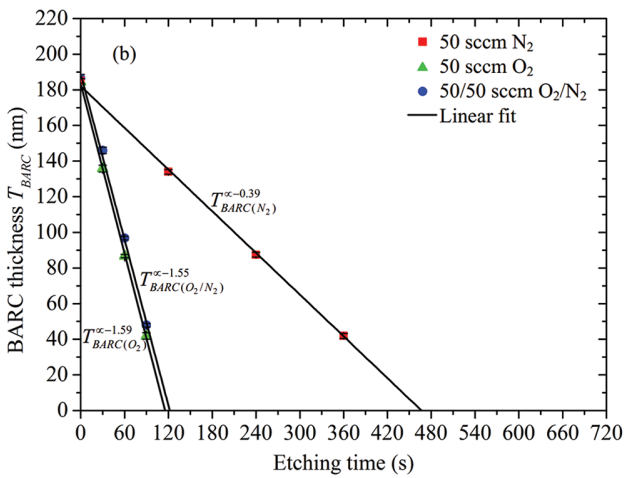

Figure 3. Thickness measurement of a) PR and b) BARC layers etched in various gas plasmas versus etching time at wafer level, $13 \mathrm{mTorr}$, and $25 \mathrm{~W}$. The linear fit curves are associated with the slopes of the lines. 
Table 1. Etching rates of PR (PFI88 1:1 PGMEA) and BARC (AZ BARLi II 200) in different gas plasmas obtained at wafer level, 13 mTorr, and $25 \mathrm{~W}$.

\begin{tabular}{lcc}
\hline $\begin{array}{l}\text { Plasma } \\
\text { etching }\end{array}$ & $\begin{array}{c}\text { PFI88 1:1 PGMEA } \\
{\left[\mathrm{nm} \mathrm{min}^{-1}\right]}\end{array}$ & $\begin{array}{c}\text { AZ BARLi II 200 } \\
{\left[\mathrm{nm} \mathrm{min} \text { m }^{-1}\right]}\end{array}$ \\
\hline $50 \mathrm{sccm} \mathrm{N} \mathrm{N}_{2}$ & 17.4 & 23.4 \\
$50 \mathrm{sccm} \mathrm{O} \mathrm{O}_{2}$ & 86.4 & 95.4 \\
$50 / 50 \mathrm{sccm} \mathrm{O}_{2} / \mathrm{N}_{2}$ & 91.2 & 93.0 \\
\hline
\end{tabular}

in maleic anhydride units and nitrogen atoms in N-substituted maleimide units, without the hydrocarbon rings. As a result, the concentration of oxygen atoms in the BARC is much higher than in the PR, i.e., $39.8 \%$ versus $13.3 \%$. In the literature, the relation between etching selectivity and PR structure has been repeatedly investigated. An increase in the number of oxygen atoms in monomer units was reported to increase the etching rates of metal-free organic materials. ${ }^{[28]}$ Using various dry etching techniques such as argon ion-beam, oxygen ionbeam, and oxygen plasma etching, Gokan et al. investigated the etching rates of various resist materials. They found that the measured etching rates are proportional to the " $N /\left(N_{\mathrm{c}}-N_{\mathrm{o}}\right)$ factor," where $N, N_{\mathrm{c}}$, and $N_{\mathrm{o}}$ are the total number of atoms, the number of carbon atoms, and the number of oxygen atoms in a monomer unit, respectively. ${ }^{[28]}$ In the presence of oxygen atoms, the carbon atoms in polymers are volatized easily during the plasma etching. ${ }^{[29]}$ Another possible reason for the difference in the etching rates could be the presence of nitrogen atoms in the BARC material. Huang et al. reported that when polymers containing nitrogen were etched in a reducing gas plasma such as $\mathrm{N}_{2}$, the nitrogen atoms cause an increase in the etching rate, by a mechanism similar to that behind the presence of oxygen atoms in polymers. ${ }^{[30]}$

In $50 \mathrm{sccm} \mathrm{O}_{2}$ plasma, the etching rates of the PR and BARC were sharply increased compared to those in $50 \mathrm{sccm} \mathrm{N} \mathrm{N}_{2}$ plasma, though the etching rate of the BARC was still higher than that of the PR. The physical bombardment of highly energetic neutrals and ions breaks the chemical bonds of polymers, and atomic oxygen or molecular oxygen can easily react with the polymer carbon to form volatile carbon oxides. ${ }^{[31]}$ However, when using a plasma mixture of $50 / 50 \mathrm{sccm} \mathrm{O} / \mathrm{N}_{2}$, the etching rate of the PR slightly increased compared to that in the $\mathrm{O}_{2}$ plasma, whereas the etching rate of the BARC slightly decreased. We attribute this to a decreased oxygen concentration, since-because of the fixed pressure of 13 mTorr-adding nitrogen resulted in a decrease in the oxygen concentration. For the BARC, the increased etching rate caused by the $\mathrm{N}_{2}$ plasma could not compensate and as a result, the overall etching rate dropped. For the PR, we explain the only slightly altered etching rate at the decreased oxygen concentration by the fact that the aromatic rings in the molecular structure of the PR could form stable structures on the surface in the $\mathrm{O}_{2}$ plasma, thereby preventing the chemical reactions. ${ }^{[30,31]}$ In fact, the $\mathrm{N}_{2}$ plasma enhanced the physical bombardment to remove these stable structures, leading to a significant increase in the overall etching rate.

Figure 4a shows the cross-sectional HR-SEM images of periodic PR nanocolumns etched in $50 \mathrm{sccm} \mathrm{N} \mathrm{N}_{2}$ plasma at different etching times, together with sketches for illustration. After $8 \mathrm{~min}$ of etching, the PR nanocolumns were completely transferred into the underlying BARC layer, resulting in BARC nanocolumns with straight sidewalls. The diameter of the nanocolumns remained largely unchanged during the etching process, though the formation of nanosharp tips was observed. These results indicate that there was no etching in the lateral direction, which means the $\mathrm{N}_{2}$ plasma etching was likely purely by physical bombardment. Physical bombardment with high energy particles has indeed been reported to lead to the formation of nanosharp tips. ${ }^{[32]}$ The geometric parameters of these nanocolumns versus the etching times were also measured and are shown in Figure 4b. Using the etching rates of the PR and BARC as previously established in $50 \mathrm{sccm} \mathrm{N}_{2}$ plasma etching, we also calculated the remaining thickness of the BARC layer

(a)
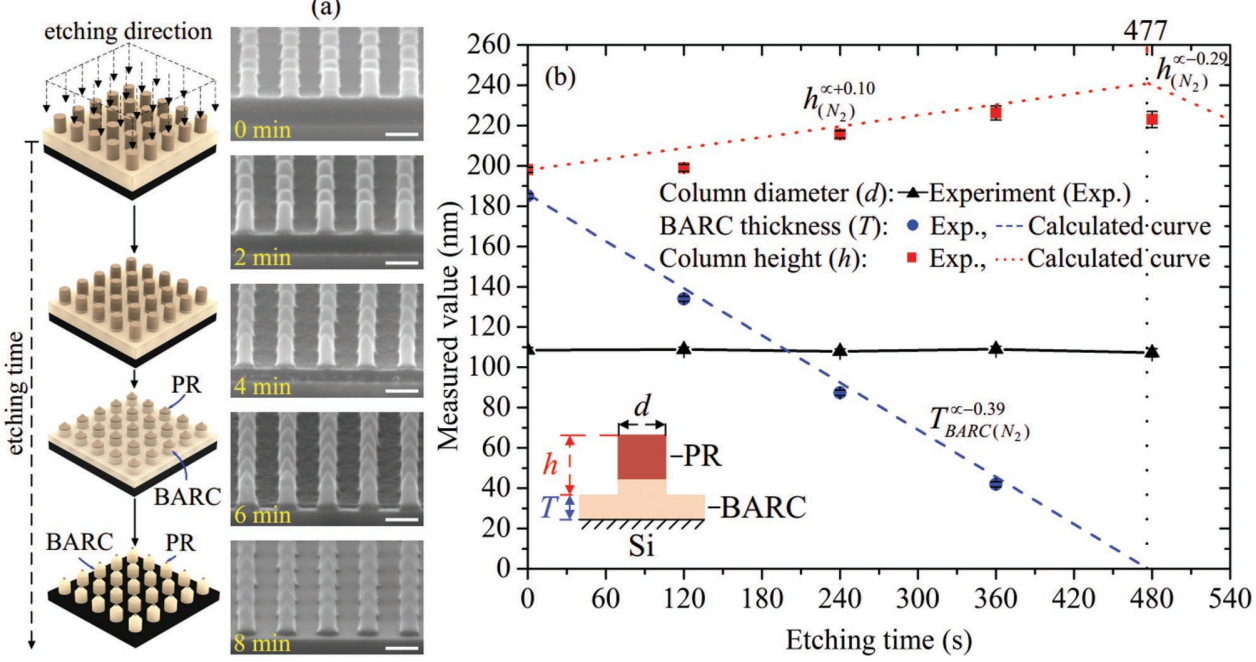

Figure 4. Patterning of periodic BARC nanocolumns using $\mathrm{N}_{2}$ plasma etching. PR nanocolumns were etched in $50 \mathrm{sccm} \mathrm{N}_{2}$ plasma at wafer level, 13 mTorr, and $25 \mathrm{~W}$, to create to BARC nanocolumns. a) Cross-sectional HR-SEM images of the nanocolumns at different etching times. Scale bars represent $200 \mathrm{~nm}$. b) Geometric measurement and calculation of the structures. 
and the height of the nanocolumns versus the etching time (see Equations (S7)-(S9), Supporting Information). The calculated curves showed a good agreement with the measurement results. The height of the nanocolumns increased with the increasing etching time because the etching rate of the PR is lower than that of the BARC. The rate of increase was $0.10 \mathrm{~nm} \mathrm{~s}^{-1}$, in accordance with the difference in etching rates of the PR $\left(0.29 \mathrm{~nm} \mathrm{~s}^{-1}\right)$ and the BARC $\left(0.39 \mathrm{~nm} \mathrm{~s}^{-1}\right)$. After a $477 \mathrm{~s}$ etching time, the BARC layer was completely etched, after which time the height of the nanocolumns decreased with the rate of the PR since the PR still remained on the top of the nanocolumns. The differences between the measurement results and the calculation results could be explained by the errors in the height measurement using the HR-SEM as well as the formation of the nanosharp tips which had not been taken into account.

We also investigated the etching of PR nanocolumn arrays in $50 \mathrm{sccm} \mathrm{O}$ plasma, and in the mixture plasma of $50 / 50 \mathrm{sccm} \mathrm{O} \mathrm{O}_{2} / \mathrm{N}_{2}$. Figure $5 \mathrm{a}, \mathrm{b}$ shows the cross-sectional HR-SEM images of the PR nanocolumn arrays etched in the $\mathrm{O}_{2}$ plasma and $\mathrm{O}_{2} / \mathrm{N}_{2}$ plasma at different etching times, respectively. For both etching processes, it was observed that the PR nanocolumns were transferred into the BARC layer, while simultaneously shrinking in diameter. Well-defined nanocolumns with vertical sidewalls were again obtained, although this time without the formation of nanosharp tips. We can explain the results as follows. The presence of oxygen probably broke chemical bonds in the nanocolumn tips due to chemical reactions. The shrinking of the nanocolumns in the $\mathrm{O}_{2}$ plasma was slightly faster than that in the mixture plasma due to the higher etching rate of the BARC obtained in a pure oxygen plasma. After $150 \mathrm{~s}$ etching, the PR nanocolumns were shrink etched into BARC nanocolumns with diameters of $24.7 \pm 3 \mathrm{~nm}$ (in $50 \mathrm{sccm} \mathrm{O} \mathrm{O}_{2}$ plasma) and $27.7 \pm 2.2 \mathrm{~nm}$ (in 50/50 $\mathrm{sccm} \mathrm{O} /$ $\mathrm{N}_{2}$ plasma) as shown in Figure $5 \mathrm{c}, \mathrm{d}$, respectively. Similar calculations of the remaining BARC thickness and the nanocolumn height were performed, based on the measured etching rates of the PR and BARC in $50 \mathrm{sccm} \mathrm{O} \mathrm{O}_{2}$ and 50/50 $\mathrm{sccm} \mathrm{O} \mathrm{O}_{2} / \mathrm{N}_{2}$ plasmas (see Equations (S10)-(S17), Supporting Information). The calculated values show a good fit with the measured values. A significant difference in the nanocolumn height between the measured values and the calculated values after $120 \mathrm{~s}$ etching in the $\mathrm{O}_{2}$ plasma was observed. This could be explained by a rapid breakage of molecular bonds in the tips, and bending of the nanocolumns due to a higher etching rate which resulted in narrower-i.e., smaller diameter-nanocolumns (see discussion of Figure 6). Highly vertical sub-30 nm BARC nanocolumns with aspect ratios as high as 5.3 were achieved in $50 / 50 \mathrm{sccm} \mathrm{O} \mathrm{O}_{2} / \mathrm{N}_{2}$ plasma after $150 \mathrm{~s}$ etching.

The uniformity of the BARC nanocolumn arrays fabricated by using different gas plasma etchings was also investigated.

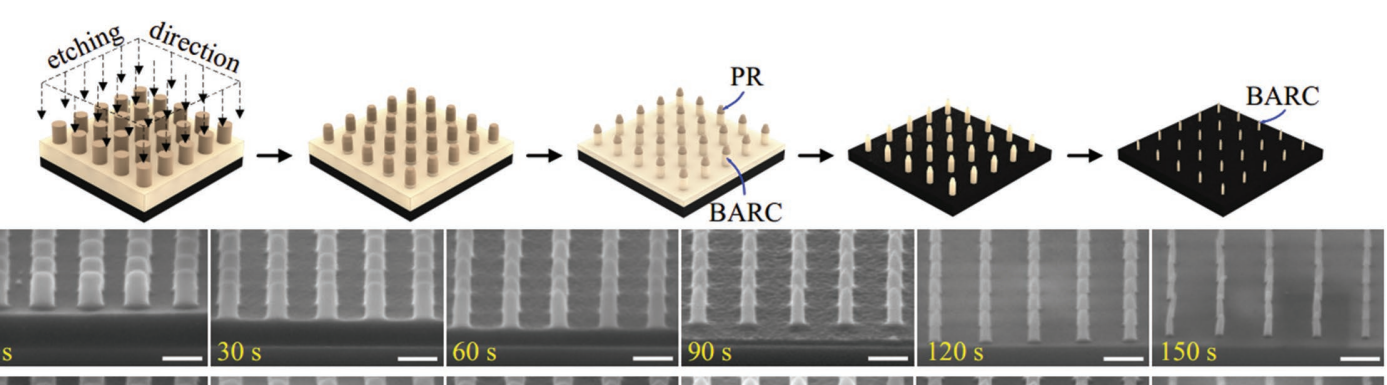

(b)
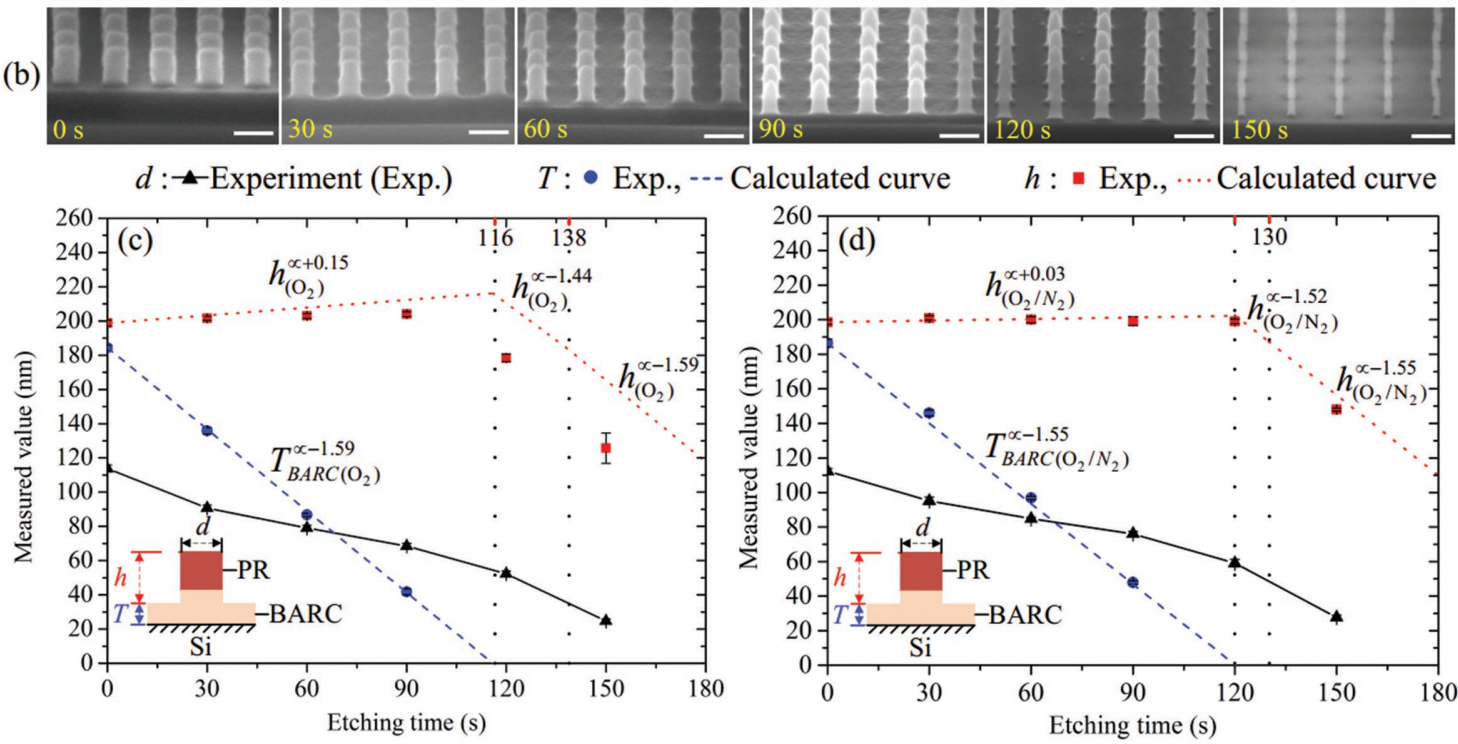

Figure 5. Patterning of periodic BARC nanocolumns using $\mathrm{O}_{2}$ or $\mathrm{O}_{2} / \mathrm{N}_{2}$ plasma etchings. PR nanocolumns were etched in a) 50 sccm $\mathrm{O}_{2}$ plasma or b) $50 / 50 \mathrm{sccm} \mathrm{O} \mathrm{O}_{2} / \mathrm{N}_{2}$ plasma at wafer level, $13 \mathrm{mTorr}$, and $25 \mathrm{~W}$. Cross-sectional HR-SEM images of the nanocolumns at different etching times at the etching parameters in (a) and (b). Scale bars represent $200 \mathrm{~nm}$. c,d) Geometric measurement and calculation of the structures obtained in (a) and (b), respectively. 


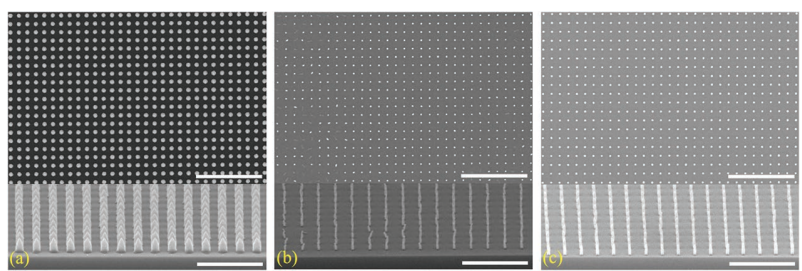

Figure 6. Top-view (scale bar: $2 \mu \mathrm{m}$ ) and cross-sectional (scale bar: $1 \mu \mathrm{m}$ ) HR-SEM images of BARC nanocolumn arrays fabricated by using different gas plasmas: a) $50 \mathrm{sccm} \mathrm{N}$ for $8 \mathrm{~min}$, b) $50 \mathrm{sccm} \mathrm{O}_{2}$ for $150 \mathrm{~s}$, and c) $50 / 50 \mathrm{sccm} \mathrm{O} / \mathrm{N}_{2}$ for $150 \mathrm{~s}$, at wafer level, $13 \mathrm{mTorr}$, and $25 \mathrm{~W}$.

Figure 6 shows the top-view and cross-sectional HR-SEM images of the fabricated BARC arrays. Highly uniform arrays of well-fabricated vertical-oriented BARC nanocolumns were obtained for $50 \mathrm{sccm} \mathrm{N}_{2}$ and $50 / 50 \mathrm{sccm} \mathrm{O} \mathrm{O}_{2} / \mathrm{N}_{2}$ plasma etchings, as shown in Figure 6a,c respectively (see Figures S9 and S10 and Table S1, Supporting Information). Smaller BARC nanocolumns were achieved in $50 \mathrm{sccm} \mathrm{O}_{2}$ plasma etching, though the nanocolumns were bent. Moreover, various BARC nanocolumns were fallen down due to their weak adhesion with the Si surface (Figure $6 \mathrm{~b}$ ). A remark that has to be made here is that insufficient cleaning of the etching chamber could create "nanograss" surfaces caused by redeposition of materials from previous etching processes, as shown in Figure 7. Such redeposited materials could also slow down or even terminate the shrinking process, so proper chamber conditions is required (see also the Supporting Information). In addition, at the etching time-step of $30 \mathrm{~s}$, no significant influence of the corona effect on the resulting nanocolumns has been observed in our etching processes. ${ }^{[33]}$

The influence of the $\mathrm{O}_{2} / \mathrm{N}_{2}$ gas flow ratio on the mixture plasma etching was investigated by changing the $\mathrm{O}_{2}$ flow rate $\left(V_{\mathrm{O}_{2}}\right)$ while keeping the $\mathrm{N}_{2}$ flow rate at $50 \mathrm{sccm}$. PR nanocolumn arrays were shrink etched in $\mathrm{O}_{2} / \mathrm{N}_{2}$ plasma at wafer level, 13 mTorr, and $25 \mathrm{~W}$ with different gas flow ratios of $\mathrm{O}_{2} / \mathrm{N}_{2}$. The diameter and height of the nanocolumns were determined after every $30 \mathrm{~s}$ of etching time from the cross-sectional images obtained by HR-SEM, as shown in Figure 8. An increase in the $\mathrm{O}_{2}$ flow rate resulted in an enhanced shrinking of the nanocolumns, especially at $\mathrm{O}_{2}$ flow rates above $10 \mathrm{sccm}$ (Figure 8a). At a $10 \mathrm{sccm} \mathrm{O}_{2}$ flow rate, even after $180 \mathrm{~s}$ etching the column diameter remained larger than $60 \mathrm{~nm}$. For $\mathrm{O}_{2}$ flow rates of $20 \mathrm{sccm}$ or above, the decrease in the column diameter was faster than at a $10 \mathrm{sccm} \mathrm{O}_{2}$ flow rate. Moreover, this decrease in the column diameter was almost independent of $\mathrm{O}_{2}$ flow rate, up to an etching time of $120 \mathrm{~s}$. When increasing the etching time by another 30 to $150 \mathrm{~s}$, differences in shrinking were observed. The diameter of the nanocolumns significantly decreased from $\approx 58$ to $\approx 38 \mathrm{~nm}\left(V_{\mathrm{O}_{2}}=40 \mathrm{sccm}\right)$, or from $\approx 59$ to $\approx 28 \mathrm{~nm}\left(V_{\mathrm{O}_{2}}=50 \mathrm{sccm}\right)$, whereas the column diameter only slightly decreased from $\approx 65$ to $\approx 55 \mathrm{~nm}$ for both the $\mathrm{O}_{2}$ flow rates of 20 and $30 \mathrm{sccm}$. Etching these nanocolumns for another $30 \mathrm{~s}$ also resulted in sub-30 nm nanocolumns, though the column height was significantly decreased (Figure 8b). Beyond a $150 \mathrm{~s}$ etching time, the remaining BARC layer was etched away completely while the nanocolumn was etched from the top, thus shortening the nanocolumns. The most efficient etching recipe for shrinking was using the mixture plasma of $50 / 50 \mathrm{sccm} \mathrm{O}_{2} / \mathrm{N}_{2}$ for $150 \mathrm{~s}$ where sub-30 nm BARC nanocolumns were obtained at the highest aspect ratio of 5.3. Using $10 / 50 \mathrm{sccm} \mathrm{O} \mathrm{O}_{2} / \mathrm{N}_{2}$ plasma to continuously shrink these nanocolumns at a lower rate could possibly lead to even narrower nanocolumns, though we would then expect a slight decrease in the uniformity of the nanocolumn distribution.

\subsection{Patterning BARC Nanocolumns at a Particular Diameter}

To fabricate BARC nanocolumn arrays at a particular diameter, we combined the etching in the mixture plasma of $\mathrm{O}_{2} / \mathrm{N}_{2}$ with the $\mathrm{N}_{2}$ plasma, as shown in Figure 9. The PR nanocolumns were first shrink etched in $50 / 50 \mathrm{sccm} \mathrm{O}_{2} / \mathrm{N}_{2}$ plasma for $60 \mathrm{~s}$ to produce nanocolumns at a diameter of $81.2 \pm 1.4 \mathrm{~nm}$. These nanocolumns were subsequently etched in $50 \mathrm{sccm} \mathrm{N}_{2}$ plasma for $4 \mathrm{~min}$ to remove the remaining BARC layer $(96 \pm 2 \mathrm{~nm})$ but without changing the nanocolumn diameter $(80.9 \pm 1.6 \mathrm{~nm})$. We also observed the formation of nanosharp tips caused by etching in the $\mathrm{N}_{2}$ plasma. Importantly, the values predicted by calculation showed a good match with the measurement results, indicating that a similar design recipe could be followed for the fabrication of any particular nanocolumn diameters (see Equations (S18)-(S21), Supporting Information). When etching in $50 / 50 \mathrm{sccm} \mathrm{O} \mathrm{O}_{2} / \mathrm{N}_{2}$ plasma, the nanocolumn height slightly increased at a rate of $+0.03 \mathrm{~nm} \mathrm{~s}^{-1}$, while the BARC thickness sharply decreased with the rate of $-1.55 \mathrm{~nm} \mathrm{~s}^{-1}$ (see Figure $5 \mathrm{~d}$ ). On the other hand, when etching in $50 \mathrm{sccm} \mathrm{N}_{2}$ plasma, the nanocolumn height strongly increased at a rate of $+0.10 \mathrm{~nm} \mathrm{~s}^{-1}$,
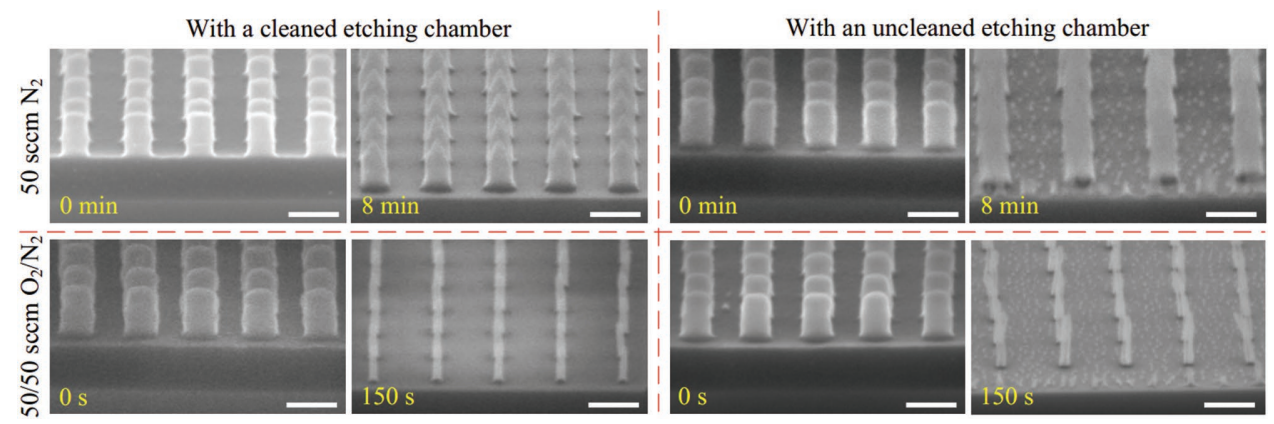

Figure 7. Comparison of the etching results obtained in a cleaned etching chamber and in an uncleaned etching chamber. Scale bars represent $200 \mathrm{~nm}$. Nanograss was formed when etching in the uncleaned chamber due to the redeposition of residual materials deposited on the chamber wall from the previous etching. 

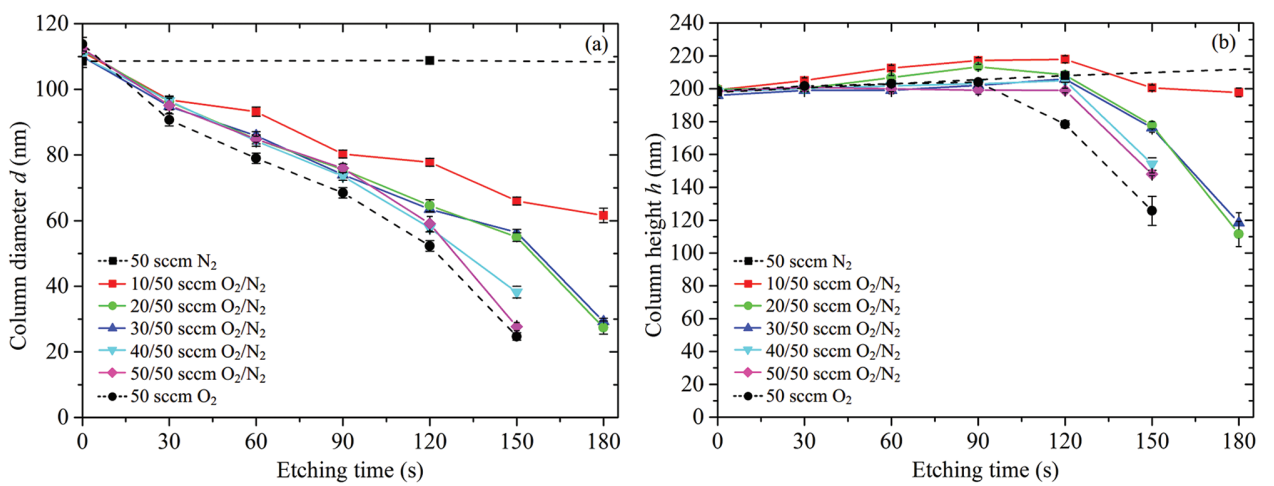

Figure 8. Measurement of a) the diameter and b) the height of the PR nanocolumns shrink etched in $\mathrm{O}_{2} / \mathrm{N}_{2}$ plasma versus etching time. The etching was conducted at wafer level, $13 \mathrm{mTorr}$, and $25 \mathrm{~W}$ with different $\mathrm{O}_{2} / \mathrm{N}_{2}$ gas flow ratios.

while the thickness of the remaining BARC slowly decreased at a rate of $-0.39 \mathrm{~nm} \mathrm{~s}^{-1}$ (see Figure $4 \mathrm{~b}$ ). We thus expect that this etching combination will enable us to easily fabricate well-defined periodic BARC nanocolumns that have vertical sidewalls and diameters ranging from $\approx 110 \mathrm{~nm}$ to sub-30 $\mathrm{nm}$.

For the shrink-etching process, the shrinkage of the nanocolumn diameter was mostly caused by oxygen plasma (Figure 5c). Minor effects have been observed when adding $\mathrm{N}_{2}$ plasma (Figure $5 \mathrm{~d}$ ). Therefore, upon using another i-line photoresist, the shrinkage of the PR nanocolumns in oxygen plasma needs to be reconducted, which can then be used as a new reference. In addition, our study revealed that for the highest uniformity in nanocolumn height the etching rate of the photoresist is close to that of BARC (Table 1). Therefore, in case of use of another resist, the flow rate ratio of oxygen to nitrogen needs to be adjusted to obtain this condition of highest uniformity on the complete specimen area. Finally, our technique is not limited to the manufacturing of nanocolumns. Using our technique, we also demonstrated the shrinkage control of PR nanolines of $90 \mathrm{~nm}$ to BARC nanolines of $45 \mathrm{~nm}$ in width (see Figure S11, Supporting Information).

\section{Conclusion}

In summary, we have successfully demonstrated a robust fabrication method that enables highly uniform large-footprint fabrication of vertical-oriented sub-30 $\mathrm{nm}$ periodic BARC nanocolumns without the need for a deep-UV laser illuminating source. Combining $\mathrm{O}_{2} / \mathrm{N}_{2}$ plasma etching with $\mathrm{N}_{2}$ plasma etching upon varying the etching times allowed us to fabricate $3 \times 3 \mathrm{~cm}^{2}$ arrays of BARC nanocolumns having different diameters ranging from sub-30 $\mathrm{nm}$ to $\approx 110 \mathrm{~nm}$, and with high control ability. Our fabrication method combines DTL using a monochromatic UV beam (365 nm wavelength) with plasma etching techniques that are simple in operation, hence making it suitable for large-area batch production at low cost. We believe the sub-30 $\mathrm{nm}$ nanopatterning method reported in this paper will provide an enabling approach for rapid patterning of $30-110 \mathrm{~nm}$ periodic nanostructures which are needed for many applications, including optoelectronic devices, ${ }^{[34]}$ photonic sensors, ${ }^{[35]}$ biosensors, ${ }^{[36]}$ nanosieve membranes, ${ }^{[37]}$ and energy applications. ${ }^{[38]}$ In fact, elsewhere we report on the fabrication of high-density arrays of sub-15 $\mathrm{nm}$ silicon quantum dots using (a)

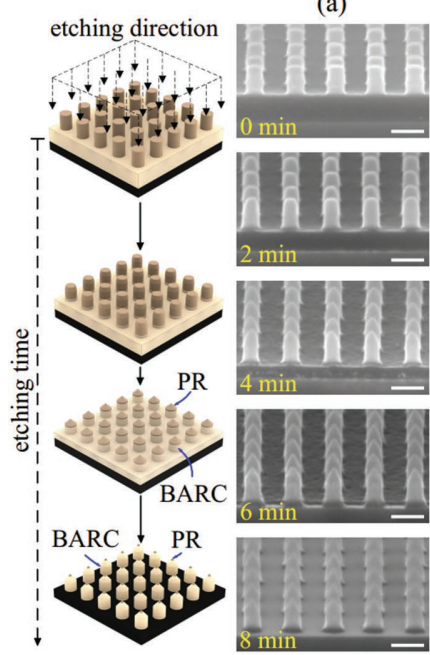

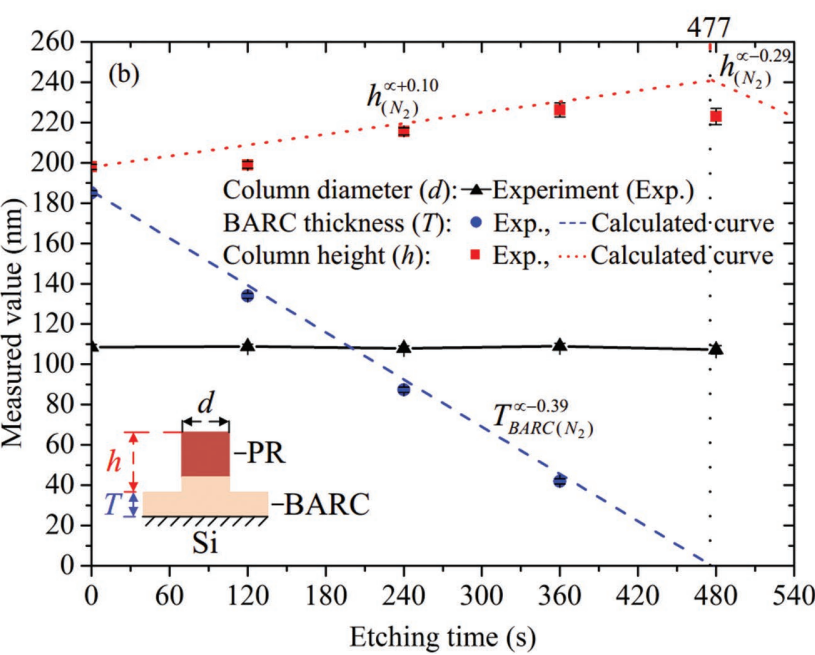

Figure 9. Patterning of periodic BARC nanocolumns at a designed diameter of $80 \mathrm{~nm}$ by combining $\mathrm{O}_{2} / \mathrm{N}_{2}$ plasma etching with $\mathrm{N}_{2}$ plasma etching. PR nanocolumns were etched in $50 / 50 \mathrm{sccm} \mathrm{O} / \mathrm{N}_{2}$ plasma, and then in $50 \mathrm{sccm} \mathrm{N} \mathrm{N}_{2}$ plasma at wafer level, $13 \mathrm{mTorr}$, and $25 \mathrm{~W}$. a) Cross-sectional HR-SEM images of the nanocolumns at different etching times. Scale bars represent $200 \mathrm{~nm}$. b) Geometric measurement and calculation of the structures. 
the combination of DTL-nanolithography and wet chemical etching steps. ${ }^{[39]}$

Research is ongoing on using this shrink-etching technique for fabricating large-area arrays of gold $(\mathrm{Au})$ nanoparticles supported on amorphous fused silica substrates. Briefly, arrays of BARC nanocolumns of different and tunable diameters are used as etching masks for pattering thin metal films into metal nanoparticles using ion beam etching (Oxford i300) (Figure S12, Supporting Information). These Au nanoparticle arrays will be used to catalyze different gas-phase reactions for investigating the influence of particle size on catalytic activity and selectivity. ${ }^{[40]}$

\section{Experimental Section}

Nanopatterning PR Nanocolumn Arrays: Four-inch Si wafers were immersed in a $1 \%$ hydrofluoric acid solution to remove the native oxide and dehydrated on a hotplate at $125^{\circ} \mathrm{C}$ for $10 \mathrm{~min}$ to remove the solvent. A BARC layer (AZ BARLi II 200, MicroChem.) was spin coated on the Si wafers at $3000 \mathrm{rpm}$ for $45 \mathrm{~s}$, followed by baking at $185^{\circ} \mathrm{C}$ for $1 \mathrm{~min}$. The Si wafers were then spin coated with positive photoresist PFI88 solution diluted 1:1 with propylene glycol methyl ether acetate (PGMEA) (Sumitomo Chemical Co., Ltd.) at $4000 \mathrm{rpm}$ for $45 \mathrm{~s}$, followed by pre-exposure baking at $90{ }^{\circ} \mathrm{C}$ for $1 \mathrm{~min}$ (Figure 1). Two exposures were implemented for each Si wafer by using displacement Talbot lithography (PhableR 100C, EULITHA) ${ }^{[23]}$ with a phase shift mask at an exposure dose of $1 \mathrm{~mW} \mathrm{~cm}$. The phase shift mask with a $3 \times 3 \mathrm{~cm}^{2}$ linear grating period of $500 \mathrm{~nm}$ made of fused silica was bought from EULITHA. The Si wafer was mounted on a vacuum chuck with a gap of $\approx 65 \mu \mathrm{m}$ from its surface to the mask. During each exposure of $45 \mathrm{~s}$, the Si wafer was displaced a DTL range of $3 \mu \mathrm{m}$ around the gap for each target cycle of every $20 \mathrm{~s}$ during the illumination of the mask by a monochromatic UV beam. The Si wafer was rotated an angle of $90^{\circ}$ relative to the mask prior to the second exposure. After two exposures, the Si wafers were postexposure baked on a hotplate at $110^{\circ} \mathrm{C}$ for $1 \mathrm{~min}$, followed by developing in the OPD4262 developer for $30 \mathrm{~s}$ and rinsing with di-iononized \& di-mineralized (DI) water to complete the fabrication of PR nanocolumn arrays.

Chamber Cleaning and Etching Implementation: The etching chamber was cleaned completely before running each plasma etching process using cleanroom tissues with acetone and then dried using a nitrogen gun to remove all the residual materials from the previous etching process. Before etching, a patterned Si wafer was broken into pieces, and one piece was selected for the HR-SEM observation of the crosssectional view. Pieces were assembled back into the wafer shape for the etching process in a TEtske machine (see Figures S3 and S4, Supporting Information). Regularly checking the selected piece using the HR-SEM was done after every etching time-step.

Plasma Etching of PR and BARC Layers: To measure the etching rates of $P R$ and $B A R C$ in various gas plasmas, Si wafers were coated with a PR PFI88 layer (4000 rpm for $45 \mathrm{~s}$, baked at $90^{\circ} \mathrm{C}$ for $1 \mathrm{~min}$ ) or with $\mathrm{AZ}$ BARLi II 200 (3000 rpm for $45 \mathrm{~s}$, baked at $185^{\circ} \mathrm{C}$ for $1 \mathrm{~min}$ ), followed by plasma etching using the TEtske machine at the wafer level, $13 \mathrm{mTorr}$, and $25 \mathrm{~W}$ (see Figures S5 and S6, Supporting Information). Three gas plasmas were used, namely $50 \mathrm{sccm} \mathrm{N}, 50 \mathrm{sccm} \mathrm{O}$, and $50 / 50 \mathrm{sccm}$ $\mathrm{O}_{2} / \mathrm{N}_{2}$, at the above etching settings with etching time-steps of $2 \mathrm{~min}$ for the $\mathrm{N}_{2}$ plasma, and $30 \mathrm{~s}$ for the $\mathrm{O}_{2}$ plasma and $\mathrm{O}_{2} / \mathrm{N}_{2}$ plasma.

Nanopatterning BARC Nanocolumn Arrays: $3 \times 3 \mathrm{~cm}^{2}$ arrays of PR nanocolumns made by DTL were subsequently etched in the TEtske machine to pattern BARC nanocolumn arrays. The etching was conducted at the wafer level, $13 \mathrm{mTorr}, 25 \mathrm{~W}$ using three gas plasmas, i.e., $50 \mathrm{sccm} \mathrm{N}$, $50 \mathrm{sccm} \mathrm{O} \mathrm{O}_{2}, 50 / 50 \mathrm{sccm} \mathrm{O} \mathrm{O}_{2} / \mathrm{N}_{2}$. The etching timestep was 2 min for $50 \mathrm{sccm} \mathrm{N}$ plasma etching, and $30 \mathrm{~s}$ for $50 \mathrm{sccm}$ $\mathrm{O}_{2}$ and $50 / 50 \mathrm{sccm} \mathrm{O}_{2} / \mathrm{N}_{2}$ plasma etching (see Figure S7, Supporting Information).
Shrink Etching with Different $\mathrm{O}_{2} / \mathrm{N}_{2}$ Gas Flow Ratios: Si wafers with patterns of $3 \times 3 \mathrm{~cm}^{2}$ PR nanocolumn arrays were shrink etched using different $\mathrm{O}_{2} / \mathrm{N}_{2}$ gas flow ratios $(10 / 50,20 / 50,30 / 50,40 / 50$, and $50 / 50 \mathrm{sccm}$ ) at wafer level, $13 \mathrm{mTorr}$, and $25 \mathrm{~W}$ with an etching time-step of $30 \mathrm{~s}$ (see Figure S13, Supporting Information).

Characterization: The HR-SEM images were all taken using an FEI Sirion microscope at a $5 \mathrm{kV}$ acceleration voltage and a spot size of 3 . The cross-sectional HR-SEM images were obtained at an angle of $20^{\circ}$ with tilt correction applied. Each error bar was calculated from the standard deviation of at least five measurements.

\section{Supporting Information}

Supporting Information is available from the Wiley Online Library or from the author.

\section{Acknowledgements}

This work was supported by the Netherlands Center for Multiscale Catalytic Energy Conversion (MCEC), and the Netherlands Organisation for Scientific Research (NWO) Gravitation program funded by the Ministry of Education, Culture and Science of the government of the Netherlands.

Received: October 14, 2016

Revised: November 14, 2016

Published online: December 21, 2016

[1] A. N. Shipway, E. Katz, I. Willner, ChemPhysChem. 2000, 1, 18.

[2] J. N. Anker, W. P. Hall, O. Lyandres, N. C. Shah, J. Zhao, R. P. Duyne, Nat. Mater. 2008, 7, 442.

[3] R. F. Pease, S. Y. Chou, Proc. IEEE. 2008, 96, 248.

[4] M Altissimo, Biomicrofluidics 2010, 4, 026503.

[5] Y. Chen, Microelectron. Eng. 2015, 135, 57.

[6] F. Watt, A. A. Bettiol, J. A. van Kan, E. J. Teo, M. B. H. Breese, Int. J. Nanosci. 2004, 4, 269.

[7] C. J. M. van Rijn, J. Microlithogr., Microfabr., Microsyst. 2006, 5, 011012.

[8] C. Lu, R. H. Lipson, Laser Photonics 2009, 4, 568.

[9] S. Y. Chou, P. R. Krauss, P. J. Renstrom, J. Vac. Sci. Technol. B 1996, 14,4129

[10] H. Schift, J. Vac. Sci. Technol. 2008, 26, 458.

[11] C. L. Haynes, R. P. van Duyne, J. Phys. Chem. B 2001, 105, 5599.

[12] X. Zhang, A. V. Whitney, J. Zhao, E. M. Hicks, R. P. van Duyne, J. Nanosci. Nanotechnol. 2006, 6, 1.

[13] H. Xu, N. Lu, D. Qi, J. Hao, L. Gao, B. Zhang, L. Chi, Small 2008, 4, 1972.

[14] A. Horrer, C. Schafer, K. Broch, A. A. Gollmer, J. Rogalski, J. Fulmes, D. Zhang, A. J. Meixner, F. Schreiber, D. P. Kern, M. Fleischer, Small 2013, 9, 4088.

[15] M. Rothschild, T. M. Bloomstein, T. H. Fedynyshyn, R. R. Kunz, V. Liberman, M. Switkes, N. N. Efremow, S. T. Palmacci, J. H. C. Sedlacek, D. E. Hardy, A. Grenville, Linc. Lab. J. 2003, 14, 221.

[16] H. H. Solak, D. He, W. Li, S. S. Gasson, S. S. Cerrina, B. H. Sohn, X. M. Yang, P. Nealey, Appl. Phys. Lett. 1999, 75, 2328.

[17] H. H. Solak, D. He, W. Li, F. Cerrina, J. Vac. Sci. Technol., B: Microelectron. Process. Phenom. 1999, 17, 3052.

[18] A. Isoyan, F. Jiang, Y. C. Cheng, F. Cerrina, P. Wachulak, L. Urbanski, J. Rocca, C. Menoni, M. Marconi, J. Vac. Sci. Technol., B 2009, 27, 2931.

[19] X. C. Yuan, D. G. Zhang, Y. Y. Sun, J. B, S. W. Zhu, J. Opt. A: Pure Appl. Opt. 2009, 11, 105104. 
[20] L. Stuerzebecher, T. Harzendorf, U. Vogler, U. D. Zeitner, R. Voelkel, Opt. Exp. 2010, 18, 19485.

[21] W. H. F. Talbot, Philos. Mag. 1836, 9, 401.

[22] L. Rayleigh, Philos. Mag. 1881, 11, 196.

[23] H. H. Solak, C. Dais, F. Clube, Opt. Exp. 2011, 19, 10686.

[24] J. R. Johnson, T. H. Gandy, G. J. Stagaman, R. J. Eakin, Proc. SPIE 1995, 2440, 562.

[25] T. S. Yang, T. Kook, J. A. Taylor, W. Josephson, M. Spak, R. R. Dammel, Proc. SPIE 1996, 2724, 724.

[26] N. Okai, Y. Sohda, Jpn. J. Appl. Phys. 2012, 51, $06 \mathrm{FB} 11$.

[27] S. Gupta, S. Jose, C. F. Lyons (Advanced Micro Devices, Inc.), US 5807790, 1998.

[28] H. Gokan, S. Esho, Y. Ohnishi, J. Electrochem. Soc. 1983, 130, 143.

[29] M. Satake, T. Iwase, M. Kurihara, N. Negishi, Y. Tada, H. Yoshida, J. Micro/Nanolithogr., MEMS, MOEMS 2013, 12, 041309.

[30] R. Huang, M. Weigand, Proc. SPIE 2008, 6923, 69232.

[31] J. A. G. Baggerman, R. J. Visser, E. J. H. Collart, J. Appl. Phys. 1994, $75,758$.
[32] Y. J. Hung, S. L. Lee, B. J. Thibeault, L. A. Coldren, IEEE J. Sel. Top. Quantum Electron. 2010, 17, 869.

[33] M. Goldman, A. Goldman, R. S. Sigmond, Pure Appl. Chem. 1985 57, 1353.

[34] S. F. Leung, Q. Zhang, F. Xiu, D. Yu, J. C. Ho, D. Li, Z. Fan, J. Phys. Chem. Lett. 2014, 5, 1479.

[35] C. Valsecchi, A. G. Brolo, Langmuir 2013, 29, 5638.

[36] B. Xiao, S. K. Pradhan, K. C. Santiago, G. N. Rutherford, A. K. Pradhan, Sci. Rep. 2016, 5, 24385.

[37] H. D. Tong, H. V. Jansen, V. J. Gadgil, C. G. Bostan, E. Berenschot, C. J. M. van Rijn, M. Elwenspoek, Nano Lett. 2004, 4, 283.

[38] C. F. Guo, T. Sun, F. Cao, Q. Liu, Z. Ren, Light Sci. Appl. 2014, 3, e161.

[39] J. G. E. Willbers, J. W. Berenschot, R. M. Tiggelaar, T. Dogan, K. Sugimura, W. G. van der Wiel, J. G. E. Gardeniers, N. R. Tas, unpublished.

[40] B. R. Cuenya, Thin Solid Films 2010, 518, 3127. 